\title{
ANTIOXIDANT POTENTIAL OF PIPERIDINE CONTAINING COMPOUNDS-A SHORT REVIEW
}

\author{
MANJUSHA R K*, SHAHEEN BEGUM, ARIFA BEGUM, BHARATHI K
}

Department of Pharmaceutical Chemistry, Institute of Pharmaceutical Technology, Sri Padmavati Mahila Visvavidyalayam (Women's University), Tirupati 517502, Andhra Pradesh, India. Email: manjurk9393@gmail.com

Received: 09 April 2018, Revised and Accepted: 14 May 2018

ABSTRACT

Piperidine is a saturated heterocyclic ring, considered as a privileged scaffold in view of its role in wide range of biological activities. Piperidine is good candidate molecule for obtaining potent antioxidant agents. The planar nature of this heterocyclic nucleus allows the introduction of substituent groups at different positions on the ring. In the present review, the antioxidant profile of piperidine containing compounds has been focused. The compounds were classified into naturally occurring piperidines, unsaturated piperidines, N-substituted piperidines, piperamides, piperanols, piperidine oximes, and hydrazides.

Keywords: Piperidine nitroxides, Substituted piperidines, Unsaturated piperidines, N-acyl substituted piperidines, Diaryl substituted piperidinones, Piperidinone oximes, Piperidine hydrazides.

(C) 2018 The Authors. Published by Innovare Academic Sciences Pvt Ltd. This is an open access article under the CC BY license (http://creativecommons. org/licenses/by/4. 0/) DOI: http://dx.doi.org/10.22159/ajpcr.2018.v11i8.26536

\section{INTRODUCTION}

Piperidine is a saturated heterocyclic secondary amine which is associated with a diverse set of biological activities such as antimicrobial, anti-inflammatory, antiviral, antimalarial, general anesthetic, antidepressant, antioxidant, antiepileptic, antitumor, anticonvulsant, and antihyperlipidemic activities [1-8]. Several clinically available drug candidates also possess this moiety in their structure (Fig. 1) [9]. Antioxidants are the compounds, which in low concentrations, are capable of either delay or inhibit the oxidative process, which occurs under the influence of atmospheric or reactive oxygen species (ROS). Antioxidants help to prevent the deleterious effects of free radicals and have been proven to be effective in oxidative stress-related diseases such as tumors, ocular related diseases, inflammation, and atherosclerosis [10-12].

Assays used for evaluating antioxidant compounds can be classified into the one which is associated with lipid peroxidation's (thiobarbituric acid assay [TBA], beta-carotene bleaching, and conjugated diene assays) and assays which are associated with electron or radical scavenging (2,2-diphenyl-1-picrylhydrazyl (DPPH), 2,2'-azinobis(3ethylbenzothiazoline-6-sulfonic acid) (ABTS), hydrogen peroxide $\left(\mathrm{H}_{2} \mathrm{O}_{2}\right)$ ferric reducing/antioxidant power (FRAP), ferric thiocyanate (FTC), and aldehyde/carboxylic acid assay) [13]. In this review article, the antioxidant activity of different piperidine containing compounds was discussed.

\section{REVIEW ON ANTIOXIDANT AND RELATED BIOLOGICAL ACTIVITIES OF PIPERIDINE CONTAINING COMPOUNDS}

\section{Naturally occurring piperidine-based compounds}

Piperine is piperidine containing an alkaloid, present in pepper extracts, i.e., Piper nigrum L. (Family Piperaceae), display strong antioxidant activity due to its ability to inhibit or quench free radicals (hydroxy and ROS). The medicinal value of piperine is very huge due to its antioxidant, antiplatelet, anti-inflammatory, antihypertensive, hepatoprotective, antithyroid, antitumor, and antiasthmatic activities. Nakatani et al. extracted various phenolic amides (Fig. 2) from pepper, which exhibited promising antioxidant properties in FTC and TBA assays. All the phenolic amides showed good antioxidant activity than $\alpha$-tocopherol at $0.01 \%$ concentration. Significant antioxidant activity was observed for amide possessing buta-1, 3-dienyl-2-methoxyphenol [14].

\section{Synthetic piperidines}

The compounds were classified into piperidine nitroxides, substituted piperidines, unsaturated piperidines, $\mathrm{N}$-acyl substituted piperidines, diarylsubstituted piperidinones, piperidinone oximes, and piperidine hydrazides.

\section{Piperidine nitroxides}

2, 2, 6, 6-Tetramethylpiperidin-1-yl) oxyl (TEMPO) and 2, 2, 6, 6-tetramethyl-4-piperidinol-N-oxyl (TEMPOL) (Fig. 3), piperidine nitroxides are potent antioxidant agents due to their ability to scavenge reactive free radicals. Trnka et al. synthesized and evaluated the antioxidant activity of TEMPOL derivatives; 2, 2, 6, 6-tetramethyl4-(5-(triphenylphosphonio) pentoxy) piperidin-1-oxy bromide (Mito TEMPOL) (Fig. 3) and hydroxylamine of Mito TEMPOL (Mito TEMPOL-H). Mito TEMPOL is readily converted to Mito TEMPOL-H by ubiquinol within mitochondria. Authors mentioned that antioxidant activity of TEMPOL might be due to its hydroxylamine and they combinedly protect mitochondria from oxidative damage [15].

In a study by Kim et al., synthesis of 3, 4, 5-trisubstituted piperidines (Fig. 4) was carried out enantioselectively, and their antioxidant activity was determined. Few of them contain a TEMPO moiety in their structure. Among all the substituted piperidines, compounds containing TEMPO moiety and hydroxy group showed promising antioxidant activity than the other compounds [16].

\section{Substituted piperidines}

Frietas et al. evaluated the in vitro antioxidant activity of $12-[(2 \mathrm{R}, 5 \mathrm{R}$, 6R)-5-hydroxy-6-methyl piperidin-2-one (iso-6-cassine; ISO) (Fig. 5) by determining the activity of glutathione-S-transferase, catalase, glutathione peroxidase, and lipid peroxidation. Anticonvulsant activity was demonstrated using pilocarpine-induced seizures in rats. ISO exhibited potent in vitro antioxidant activity and also increased the latency to the onset of seizures. Authors suggested that anticonvulsant activity of ISO was might be due to its antioxidant activity [7].

2,3-Dihydro-1H-benzo[d]imidazole moiety was introduced at the $4^{\text {th }}$ position of 3-methyl-2,6-diarylpiperidine by condensing 0-phenylenediamine and 3-methyl-2,6-diarylpiperidine-4one. The obtained compound 3'-methyl-2', 6'-diphenyl-1,3dihydrospiro[benzo[d]imidazole-2,4'-piperidine (Fig. 6) was evaluated for its antioxidant activity using DPPH assay. The compound showed significant free radical scavenging activity [17] 


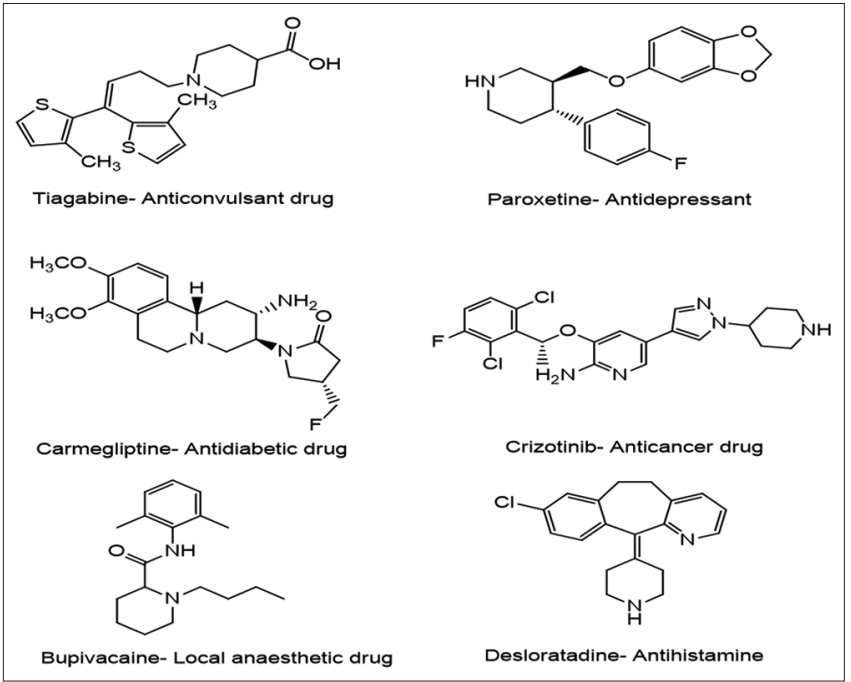

Fig. 1: Clinically available piperidine containing drugs

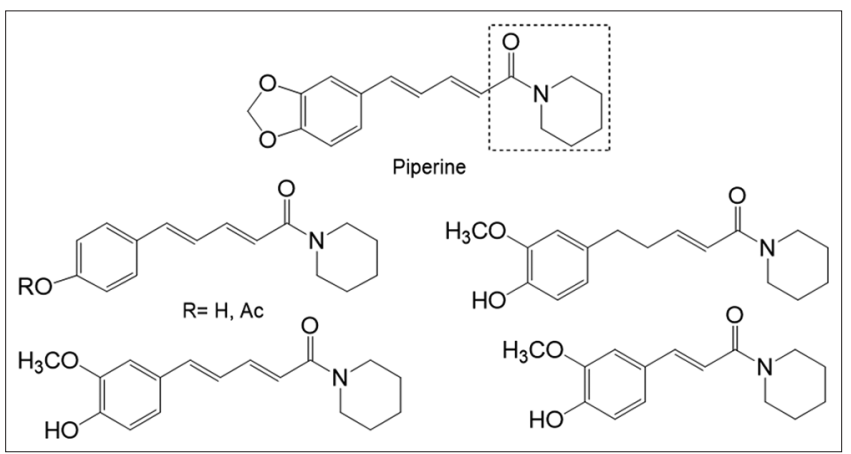

Fig. 2: Naturally occurring compounds bearing piperidine ring

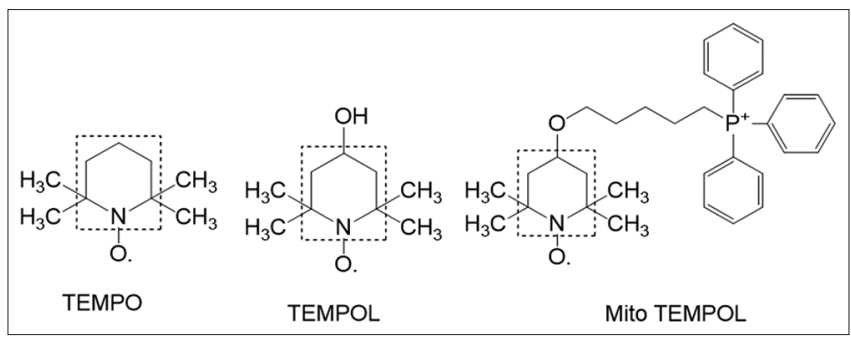

Fig. 3: Structures of 2, 2, 6, 6-Tetramethylpiperidin-1-yl) oxyl, 2, 2, 6, 6-tetramethyl-4-piperidinol-N-oxyl (TEMPOL) and Mito TEMPOL
Alexidis et al. synthesized different piperidine derivatives (Fig. 7) by introducing several substituent groups with chelating properties. Cysteamine derivatives exhibited potent antioxidant activity in the assays such as lipid peroxidation inhibitory assay, hydroxyl radical scavenging assay, and DPPH assay due to oxidizable SH group. The derivatives in which SH group was replaced by hydroxy and amine functionality, showed poor antioxidant activity. The lipid peroxidation inhibition was time and concentration dependent [18].

Kiasalari et al. prepared phenylcyclidine (PCP) and its analog 1-[1-(3-methoxyphenyl) (tetralyl)] piperidine (PCP1) (Fig. 8) by introducing phenyl cyclohexyl ring and 1-(2-methoxyphenyl)-1, 2, 3, 4-tetrahydronaphthalene ring at piperidine nitrogen. Antioxidant activity of PCP and PCP1 was evaluated using malondialdehyde, nitric oxide (NO), and superoxide dismutase (SOD) assessment and their anticonvulsant effect was studied using pentylenetetrazol-induced kindling model. The results showed that PCP1 exhibited marked antioxidant and anticonvulsant activity when compared to PCP [5].

Different derivatives of ethyl N-aryl-2, 6-dioxo-piperid-3-ene-4carboxylates (Fig. 9) were synthesized and screened for their antioxidant and antimicrobial activities. Aryl groups possessing electron donating/ withdrawing groups were introduced on the nitrogen of ethyl 2,6-dioxo1,2,3,6-tetrahydropyridine-4-carboxylate. Among the synthesized compounds, derivatives with unsubstituted phenyl ring and 4-nitro substituted phenyl ring showed highest DPPH free radical scavenging activity. Most of the compounds showed moderate antioxidant and antimicrobial activities [19].

2E,5E)-2,5-bis (3-bromo- 5-methoxy- 4-(2-(piperidin-1yl) ethoxy) benzylidene) cyclopentanone; (2E,5E)-2,5-bis (3-bromo- 5-methoxy- 4-(2-(2-methylpiperidin-1-yl) ethoxy) benzylidene) cyclopentanone; (2E,5E)-2,5-bis (3-bromo-5methoxy-4- (3-(piperidin-1-yl)propoxy) benzylidene) cyclopentanone; and (2E,5E)-2,5-bis (3-bromo-5-methoxy-4-(3-(2-methylpiperidin-1yl)propoxy)benzylidene)cyclopentanone (Fig. 10) were synthesized while preparing C5-curcuminoids. These compounds were evaluated for their in vitro antioxidant (DPPH free radical scavenging activity) and antibacterial activities. Derivatives possessing amino functionalities showed poor antibacterial activity [20].

A series of piperidine sulfonamide derivatives (Fig. 11) were synthesized by coupling of different sulfonyl chlorides with 4-(piperidin-1-yl) aniline. Antioxidant activity was evaluated using DPPH assay. Enzyme inhibitory activities were screened using butyrylcholinesterase, lipoxygenase, and acetylcholinesterase enzymes. $\mathrm{N}-[4-\{(4-$ piperidin-1-yl) acetamido) benzenesulfonamide demonstrated good scavenging activity in DPPH assay. This study also concluded that the unsubstituted derivative can be a potent molecule for the treatment of Alzheimer's disease, cancer, inflammation, and bronchial asthma [21].

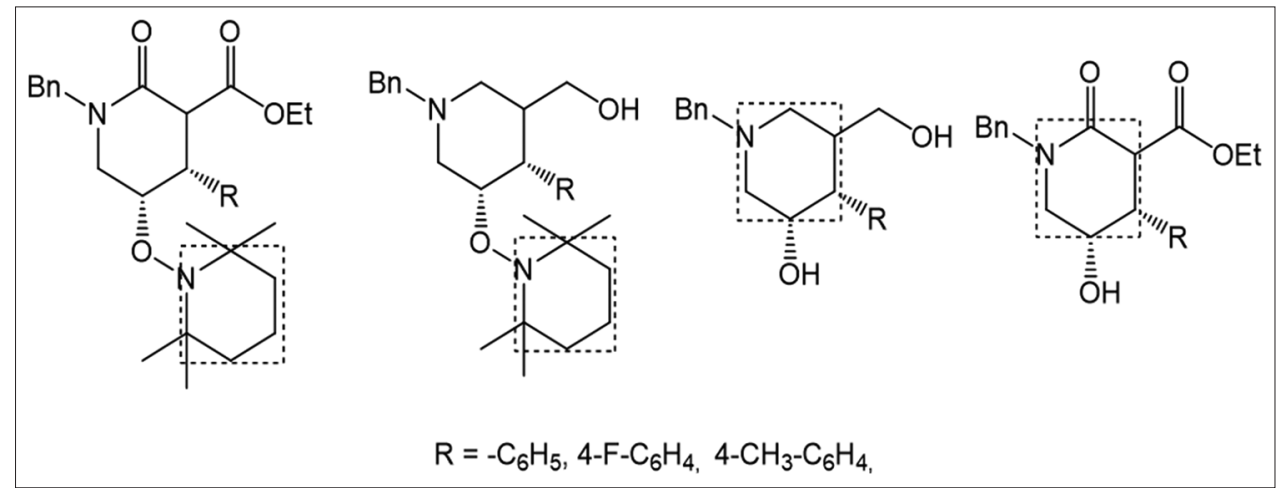

Fig. 4: 3, 4, 5-trisubstituted piperidines 


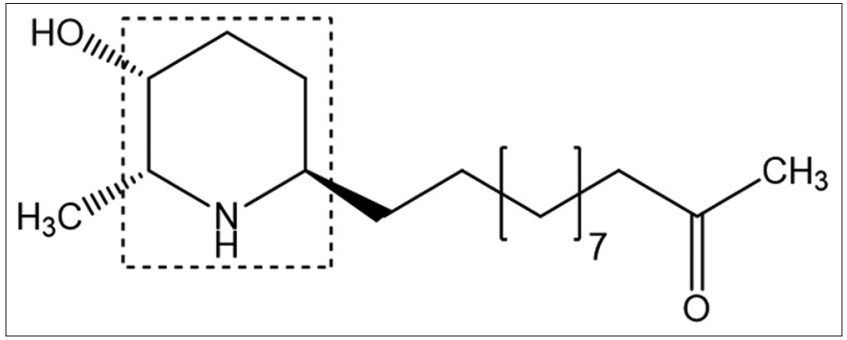

Fig. 5: Iso-6-cassine

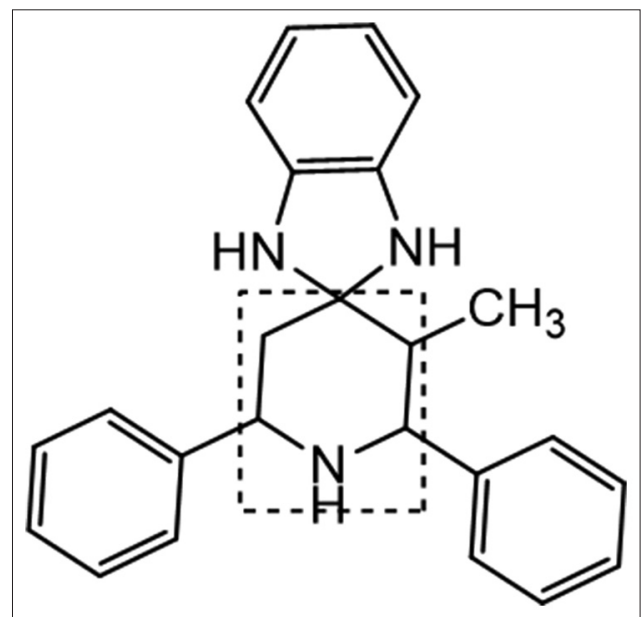

Fig. 6: Structure of 3'-methyl-2', 6'-diphenyl-1, 3-dihydrospiro[benzo[d]imidazole-2,4'-piperidine

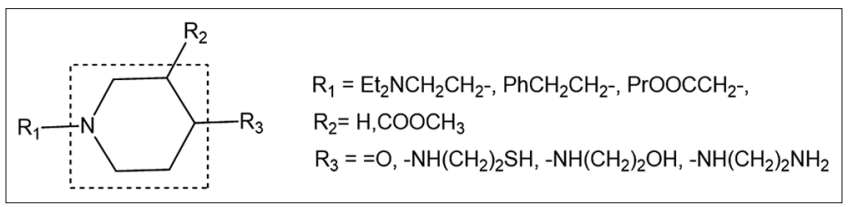

Fig. 7: N- substituted piperidines bearing cysteamine moiety

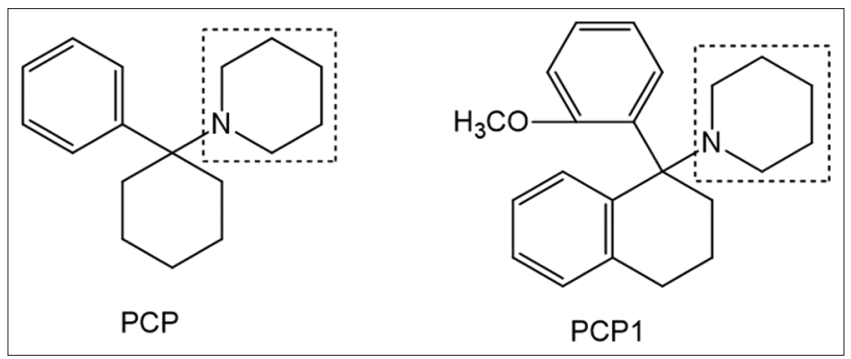

Fig. 8: Structures of phenylcyclidine (PCP) and its analog (PCP1)

A series of alkyl piperidines (Fig. 12) were synthesized using piperidine2-methanol and piperidine-2-ethanol as parent structures and screened for their antioxidant, antibacterial, and antifungal activities. It was observed that a compound possessing fluoro group at para position demonstrated good antioxidant, antibacterial, and antifungal activities. Results concluded that good antioxidant activity of the active compounds was due to the presence of halogens fused with methanol group [22].

A novel series of nitrogen-containing (piperidine, morpholine, and N-methyl piperazine) benzophenone analogs (Fig. 13) were synthesized by Mannich reaction. The compounds elicited inhibitory activity against tumor necrosis factor- $\alpha$ (TNF- $\alpha$ ), interleukin-6 (IL-6)

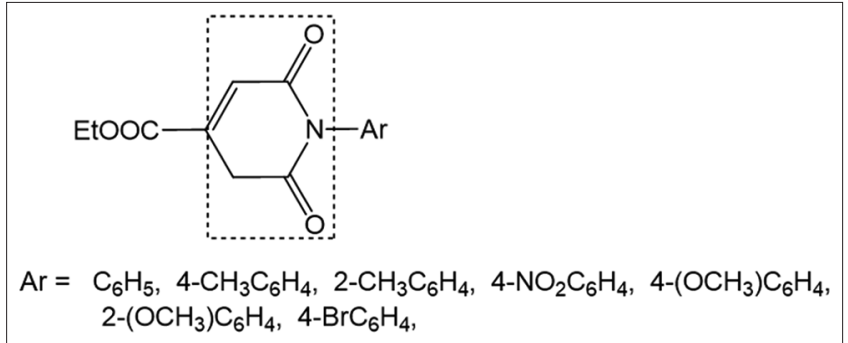

Fig. 9: Structure of ethyl N-aryl-2, 6-dioxo-piperid-3-ene-4carboxylates

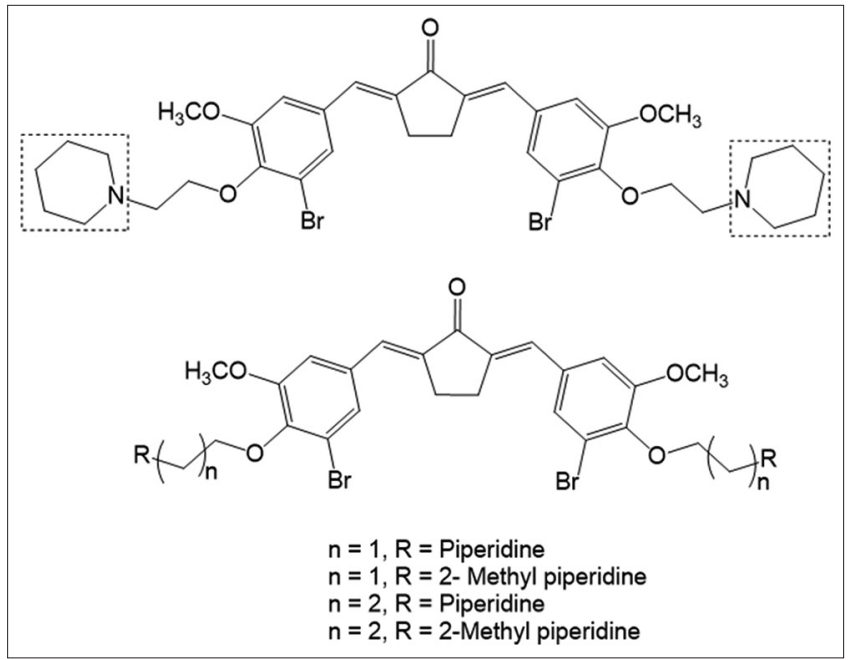

Fig. 10: N- substituted piperidines

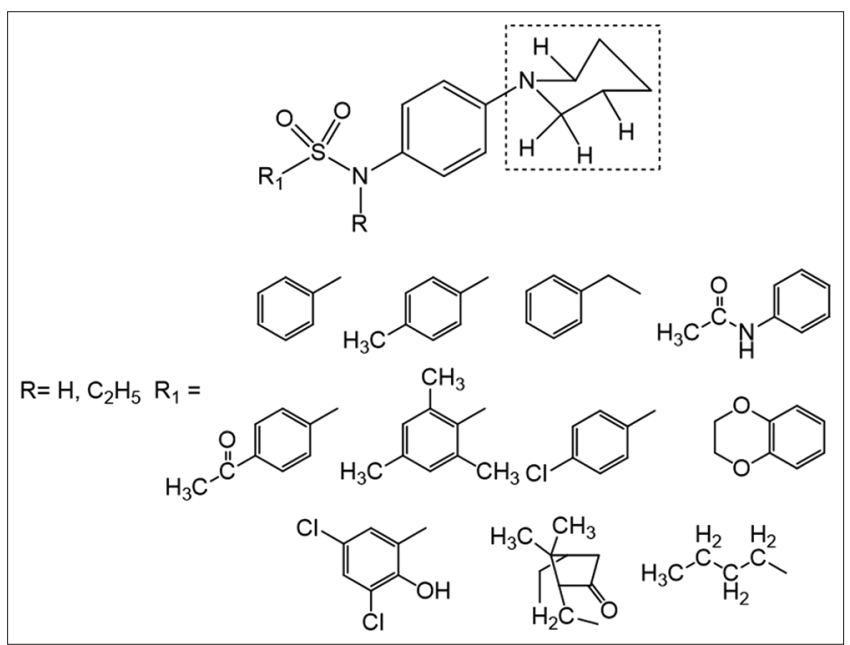

Fig. 11: Piperidine coupled sulfonamide derivatives

cytokines and found to be active in DPPH assay. Piperidine substituted compounds showed good antioxidant activity and also elicited 20$100 \%$ TNF- $\alpha$ inhibition at $10 \mu \mathrm{M}$ and $83-100 \%$ IL-6 inhibition at $10 \mu \mathrm{M}$, respectively [23].

\section{Unsaturated piperidine derivatives}

A series of piperidine containing derivatives (Fig. 14) were synthesized, where nitrogen is substituted with an acetyl group. These derivatives were screened for antioxidant (DPPH and SOD assays) and antimicrobial activities. Among all, 1-Adamantylthio derivative bearing methyl group demonstrated potent antioxidant activity (in both the assays) and antimicrobial activity [24]. 
Piperidine derivatives (unsaturated at 3 and 4 positions) (Fig. 15) were synthesized, and their antioxidant and antimicrobial activities were evaluated. Among the synthesized derivatives, derivative bearing methoxy substituent at $R_{3}$ and $R_{8}$ positions showed highest DPPH free radical scavenging activity whereas compound possessing cyano group at $\mathrm{R}_{2}$ position showed poor scavenging activity. Antibacterial activity was evaluated using agar disc diffusion method, in which compound containing trifluoromethyl group at $\mathrm{R}_{3}$ and $\mathrm{R}_{8}$ positions showed poor antibacterial activity and the compound which contains cyano group at $\mathrm{R}_{2}$ position showed potent antibacterial activity [25].

\section{$\mathrm{N}$-acyl substituted piperidines}

Piperamide derivatives (Fig. 16) were synthesized by treating different piperazine and piperidine compounds with (E)-3-(7-methoxybenzo[d] [1,3]dioxol-5-yl)acrylic acid. The compounds were evaluated for their antibacterial, antifungal (disc diffusion method), antidepressant (forced swim test and tail suspension test), antioxidant (DPPH and superoxide radical scavenging method) activities and also for their monoaminooxidase A and B inhibitory activity. Among the synthesized piperamides, the one possessing hydroxyl group on $4^{\text {th }}$ position of piperidine ring showed highest antioxidant capacity, whereas 4-phenyl substituted piperidine derivative exhibited poor antioxidant activity indicating that this substitution is unfavorable for the activity [4].

Piperamide derivatives (Fig. 17) were synthesized using different substituted cinnamic acids. The prepared compounds were evaluated for their antibacterial and antioxidant (using DPPH and $\mathrm{H}_{2} \mathrm{O}_{2}$ radical

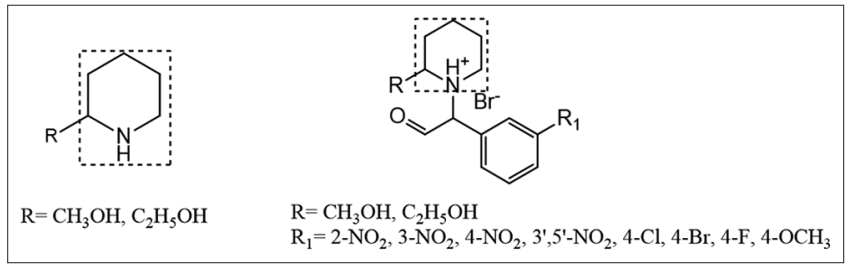

Fig. 12: Structures of alkyl piperidines

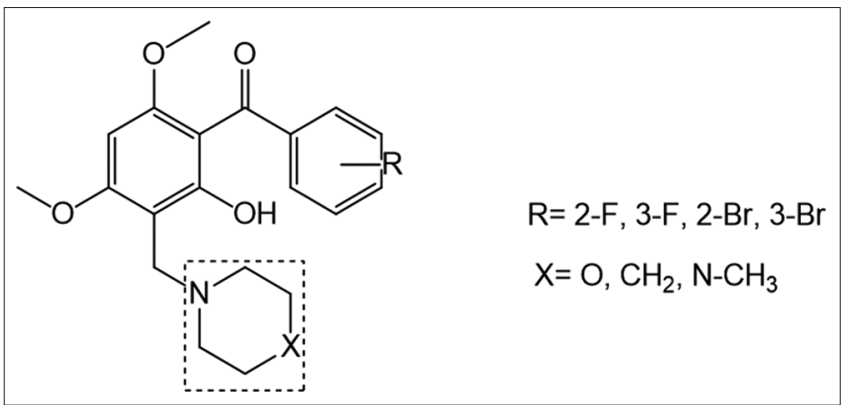

Fig. 13: Nitrogen-containing benzophenone derivatives

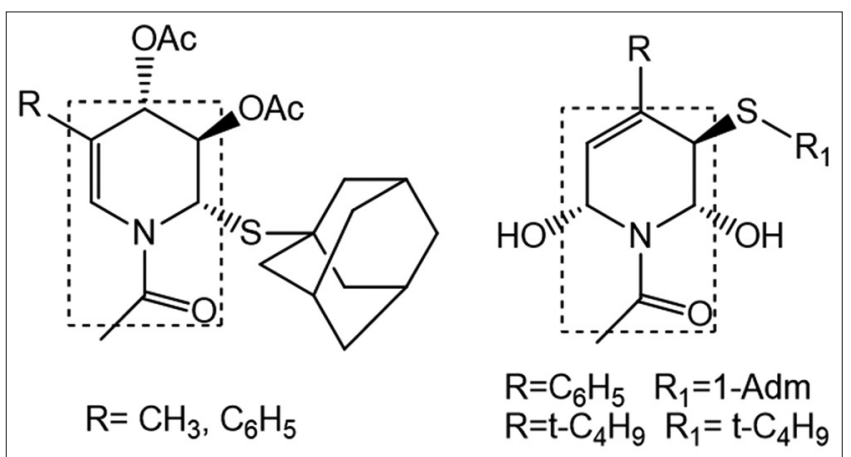

Fig. 14: N- acyl substituted piperidines (unsaturated piperidine ring) scavenging assay) activities. Significant antioxidant and antibacterial activities were noticed for methoxy containing piperamides. Among all the prepared derivatives, derivative bearing chloro group on phenyl ring demonstrated promising antifungal activity [26].

[1-(Substituted- benzoyl)-piperidin-4-yl]-(2,4-difluoro-phenyl)methanone oximes (Fig. 18) were synthesized and screened for their in vitro antioxidant (DPPH and ferrous ion chelating assays), antibacterial, and antiproliferative (MTT(3-(4, 5-dimethylthiazolyl-2)-2, 5-diphenyltetrazolium bromide) assay) activities. All the derivatives exhibited good antioxidant activity, in that; compound bearing trimethoxy substitution on phenyl ring demonstrated promising antioxidant activity in both the assays [27]

6-Fluoro-3-(piperidin-4-yl) benzo [d] isoxazole derivatives (Fig. 19) were synthesized and evaluated for their antioxidant and antimicrobial activities. Compounds were evaluated for their antioxidant efficacy in $\mathrm{H}_{2} \mathrm{O}_{2}$ and DPPH methods. Among all, derivatives with electron withdrawing groups $(\mathrm{F}, \mathrm{Cl})$ exerted good antioxidant and antimicrobial activities [28].

Piperidine conjugated benzisoxazole derivatives (Fig. 20) were synthesized and screened for their antioxidant, antibacterial, and anti-inflammatory activities. The results indicated that compounds bearing electron donating groups such as $\mathrm{OCH}_{3}, \mathrm{CH}_{3}$ groups exerted good inhibitory activity in DPPH, hydroxyl, and superoxide anion radical scavenging assays, while compounds with dinitro substituents exhibited highest antibacterial activity, compounds with nitro group

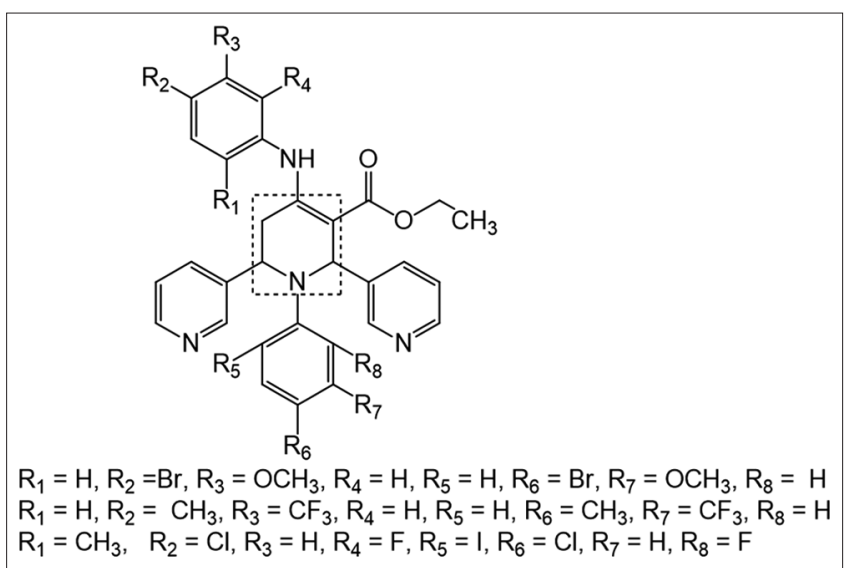

Fig. 15: Structure of piperidine derivatives which contain unsaturation at 3 and 4 positions

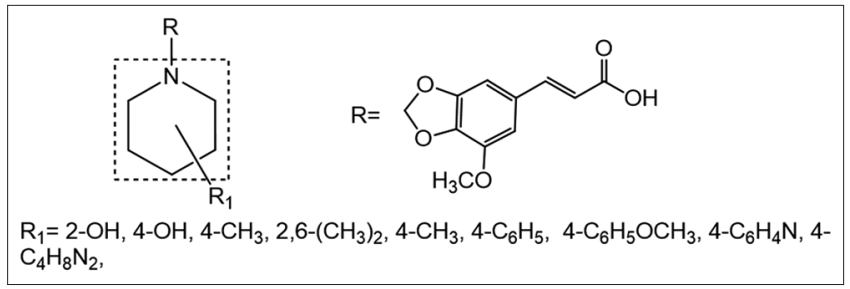

Fig. 16: $\mathrm{N}$ - acyl substituted piperidine derivatives

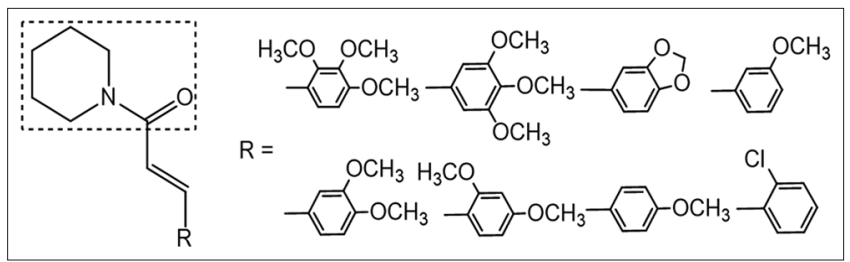

Fig. 17: Piperamide derivatives 
showed highest anti-inflammatory activity in both phospholipase A2 and lipoxygenase inhibition assays [29]. By replacing sulfonyl group with carbonyl moiety, authors further synthesized several benzisoxazole derivatives (Fig. 20) and determined their antioxidant, antibacterial, and anti-inflammatory activities.

Methyl and methoxy substituted derivatives displayed good antioxidant activity against DPPH, superoxide anion, and hydroxyl radical scavenging assays. Antibacterial activity was evaluated using disc diffusion method, in which compound with unsubstituted phenyl ring exhibited highest antibacterial activity. The derivatives containing 4-methyl or 2/4-nitro group showed good anti-inflammatory activity [30].

\section{Diaryl substituted piperidinones}

Piperidinone based compounds, especially substituted with aryl rings at $\mathrm{C}_{2}$ and $\mathrm{C}_{6}$ positions display promising antioxidant activities. Ajay et al. recently reviewed various synthetic procedures and biological activities of diaryl substituted piperidinones [31]. The antioxidant potential of 3-benzylidene-5-methyl-2,6diarylpiperidin-4-ones (Fig. 21) was studied using DPPH assay. In the tested compounds (Z)-3- (4-chlorobenzylidene) -2,6bis (4-chlorophenyl) -5-methylpiperidin-4-one and (Z)-3-(3nitrobenzylidene) -5-methyl -2,6-bis(3-nitrophenyl) piperidin-4-one (Fig. 21) were found to be more active when compared to ascorbic acid. Authors mentioned that electron-donating nature of the substituents such as hydroxy or methoxy groups on 3-arylidene-4-piperidones might be responsible for antioxidant activity [32]

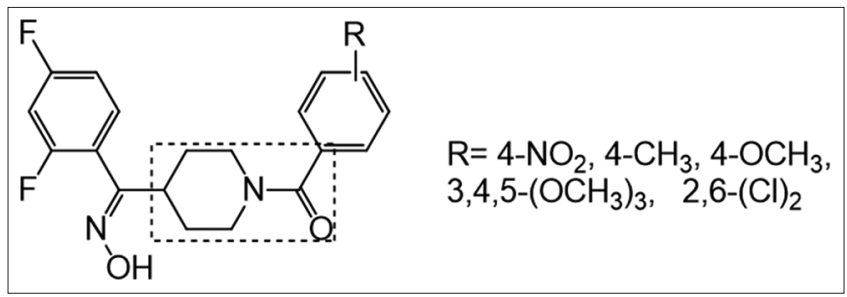

Fig. 18: $\mathrm{N}$-acyl substituted piperidine derivatives

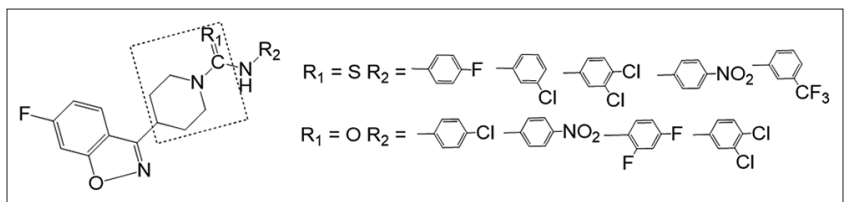

Fig. 19: 6-Fluoro-3-(piperidin-4-yl) benzo [d] isoxazole derivatives
Substituted piperidines and their respective alcohols (Fig. 22) were synthesized and evaluated for their antioxidant activity. Piperidones showed a highest antioxidant effect when compared to the isomeric alcohols [33].

A series of 4-methyl-N'-(3-alkyl-2r,6C-diaryl piperidin-4-ylidene)1,2,3-thiadiazole-5-carbohydrazides (Fig. 23) were synthesized and evaluated for their antioxidant, antimicrobial, and anticancer activities. Antioxidant activity was evaluated by DPPH, ABTS, superoxide, hydroxyl, and NO free radical scavenging assays. Compounds possessing electron donating groups (methoxy and methyl) at para position of phenyl ring exhibited significant free radical scavenging activity. Compounds possessing electron withdrawing groups such as fluoro or chloro or bromo groups at para position of phenyl ring which is attached to piperidine ring at $\mathrm{C}_{2}$ and $\mathrm{C}_{6}$ positions, displayed promising antitumor and antimicrobial activities [6].

\section{Piperidinone oximes}

A series of novel 2,6-diphenyl-3-alkylpiperidin-4-one-0-[2,4,6-tritertiary butyl cyclohexa -2-5-dienon-4-yl] oximes (Fig. 24) were synthesized, and their antioxidant and antimicrobial activities were screened. Most of the compounds were effective free radical scavengers in DPPH, superoxide, NO, ABTS, and hydroxyl assays. Compound with the electron releasing ethyl group exhibited promising antioxidant activity when compared to other compounds. Results showed that the compound possessing ethyl group at $3^{\text {rd }}$ position on piperidine moiety showed potent antimicrobial and antifungal activities [34].

Substituted piperidine oximes were synthesized and evaluated for their antioxidant (DPPH and superoxide free radical scavenging assays) and anti-inflammatory (carrageenan-induced rat paw edema) activities. 3, 3-Dimethyl 2, 6- dimethyl piperidine 4-one oxime (Fig. 25) exerted potent antioxidant activity. The active compound also displayed marked antiinflammatory activity comparable to standard drug dexamethasone [35]

Harini et al. synthesized various piperidinone oxime esters (Fig. 26) and screened in vitro antioxidant and antimicrobial activities. Results showed that antioxidant activity was (DPPH assay, ABTS radical scavenging assay, FRAP assay, and cupric ion reducing antioxidant capacity assay) enhanced when the hydroxy group was introduced on the phenyl ring. Among the synthesized compounds, compounds with electronegative fluoro or chloro or bromo group on benzoyl ester moiety showed excellent antibacterial activity whereas compound with the fluoro group exhibited significant antifungal activity [36].

Harini et al. synthesized piperidinone oxime esters by replacing 4-methoxy phenyl ring with vanillin moiety few (Fig. 27) and screened their antioxidant activity. Compounds bearing hydroxy groups on

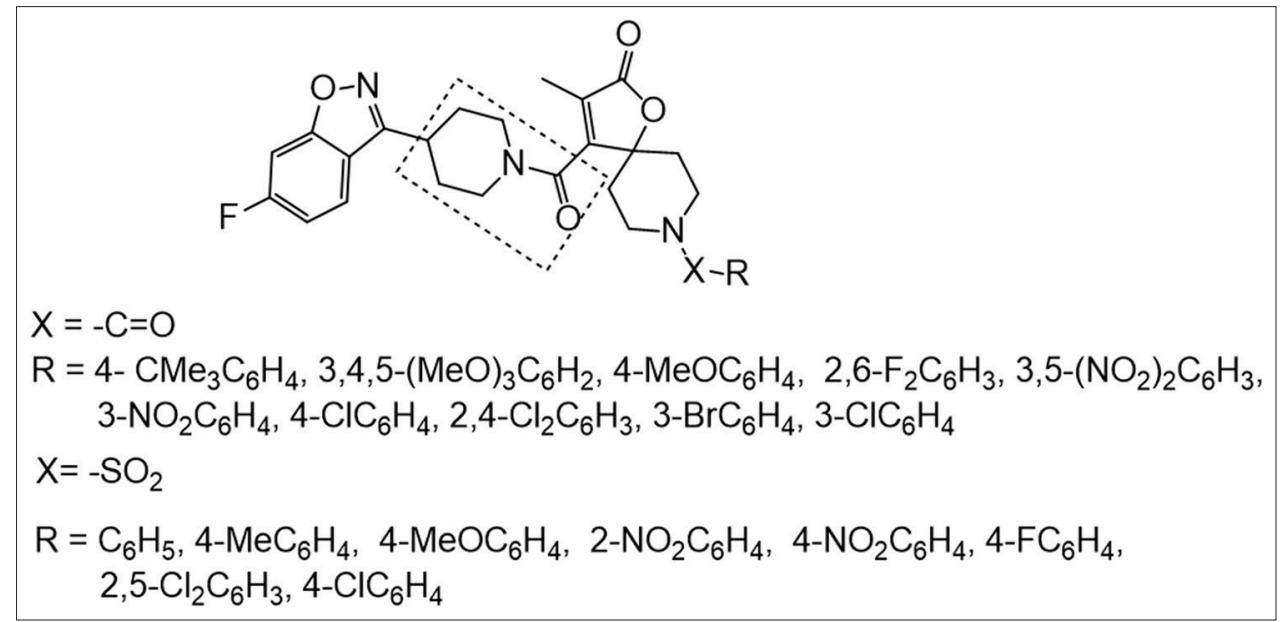

Fig. 20: Piperidine conjugated benzisoxazole derivatives 
phenyl ring demonstrated potent antioxidant activity. Compounds possessing fluoro or chloro group displayed good antibacterial and antifungal activities [37].

Further, thiazole-based piperidinone oximes were synthesized by the same research group, and their antioxidant and

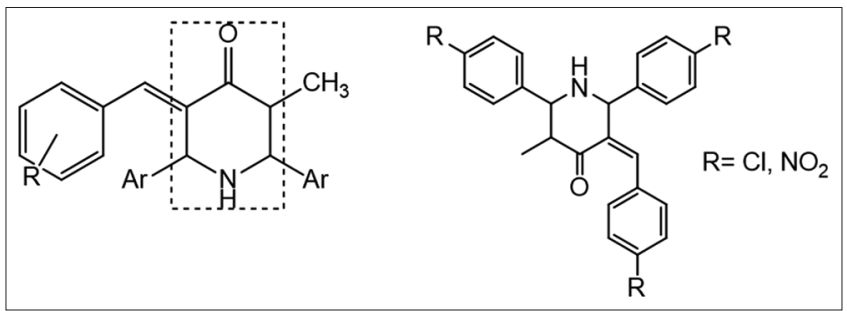

Fig. 21: Diaryl substituted piperidinones

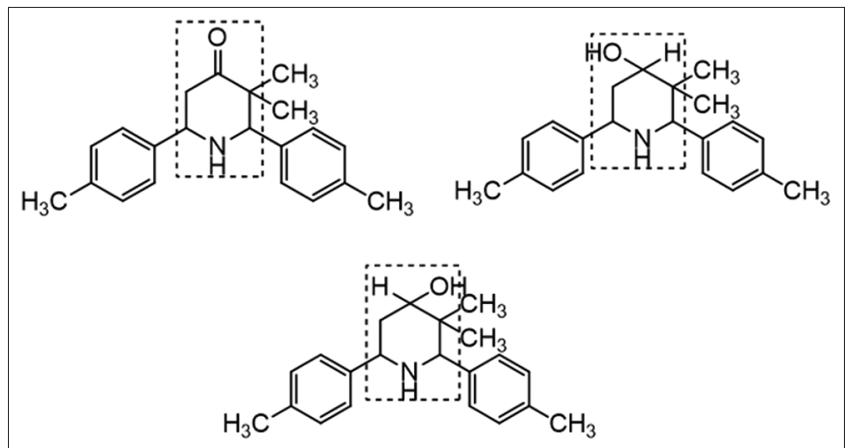

Fig. 22: Substituted piperidines

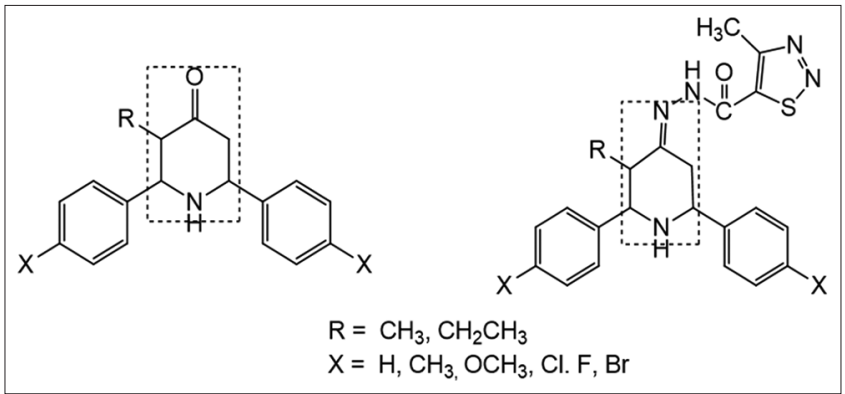

Fig. 23: Structures of 4-methyl-N'-(3-alkyl-2r, 6C-diaryl piperidin4-ylidene)-1,2,3-thiadiazole-5-carbohydrazides

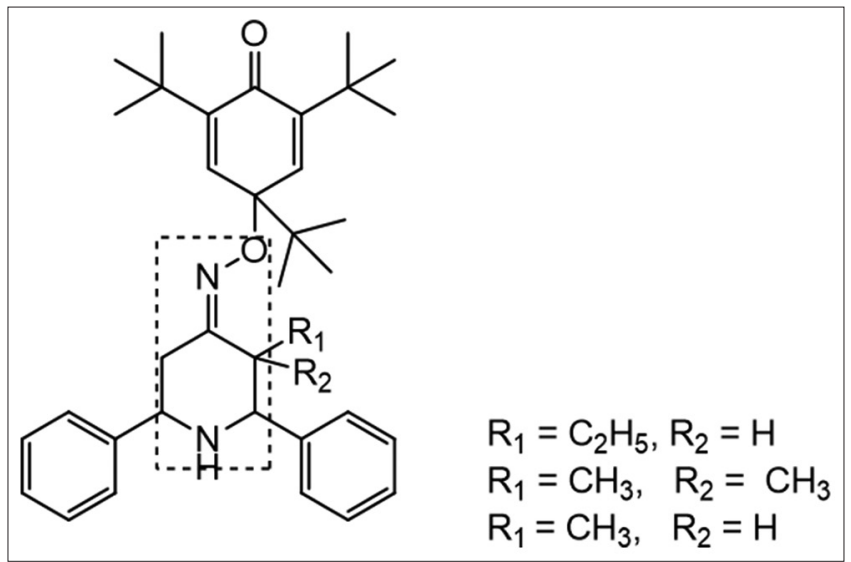

Fig. 24: Substituted 2,6-diphenyl-3-alkylpiperidin-4-one-0[2,4,6- tritertiary butyl cyclohexa -2-5-dienon-4-yl] oximes antimicrobial activities were evaluated. Among all, 2,6-bis(4hydroxy-3- methoxyphenyl) -1-methylpiperidin -4-one 0-(2-(2-(4hydroxy- 3-methoxybenzylidene) hydrazinyl) thiazol4-yl) oxime

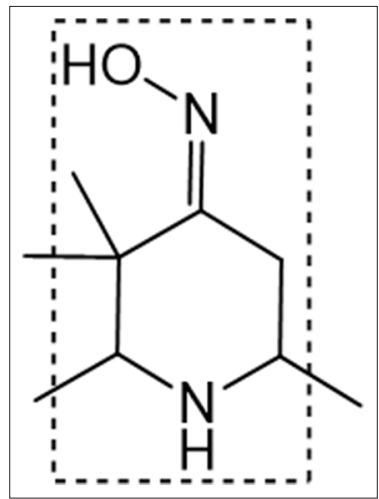

Fig. 25: Substituted piperidine oxime structures

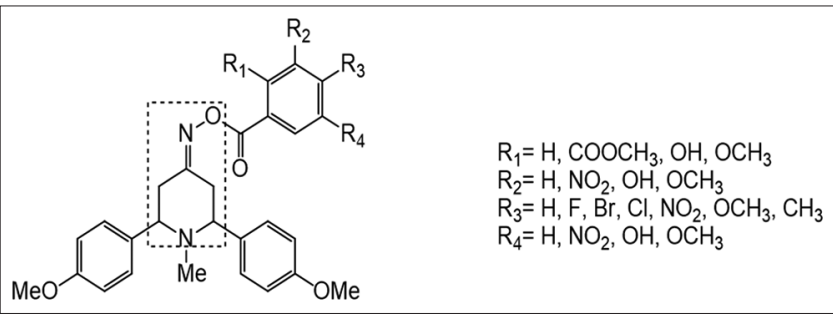

Fig. 26: Piperidinone oxime esters

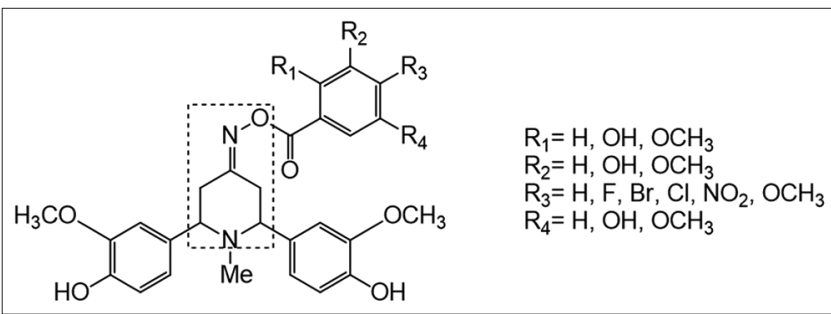

Fig. 27: Piperidinone oxime esters

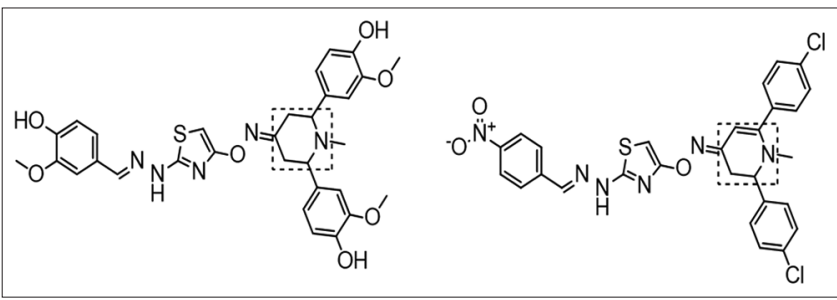

Fig. 28: Thiazole-based piperidinone oximes

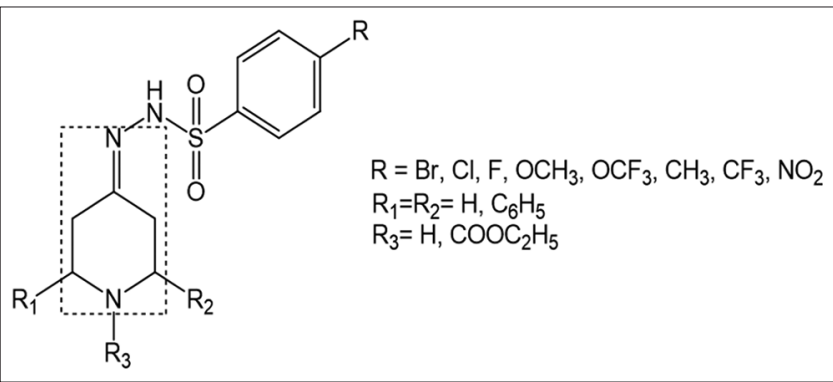

Fig. 29: Sulfonyl hydrazones bearing piperidine ring 


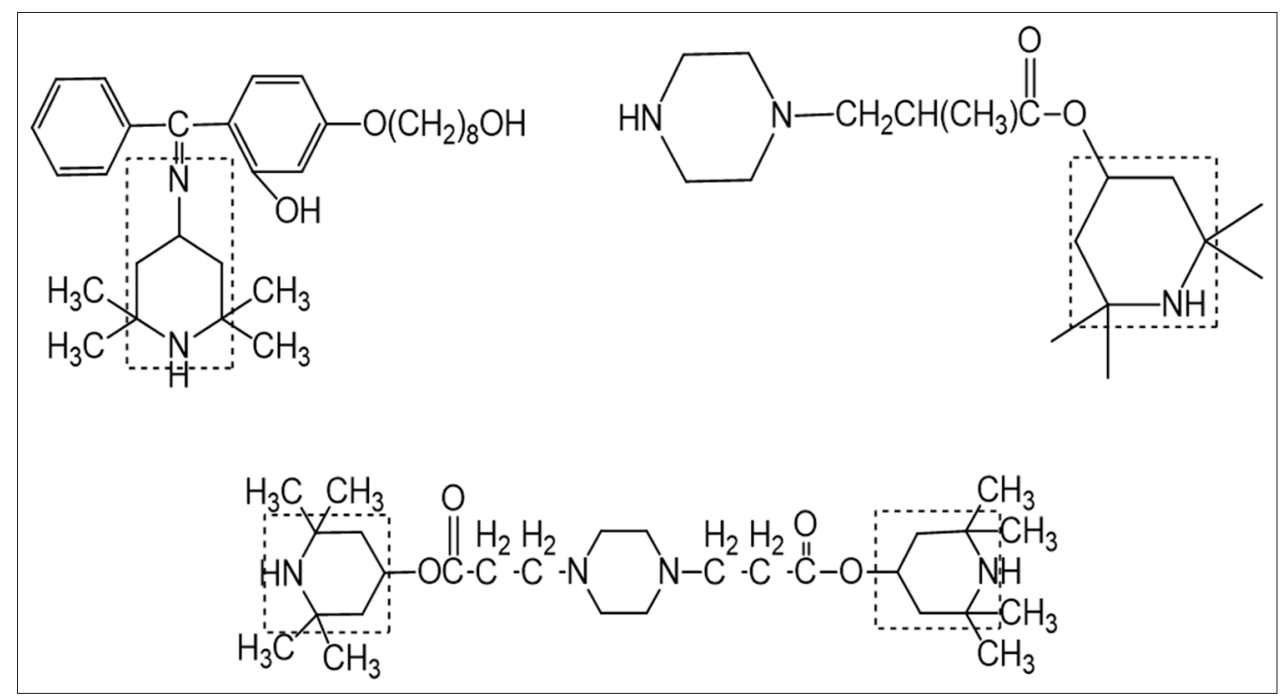

Fig. 30: Imine substituted piperidine derivatives

(Fig. 28) displayed promising antioxidant activity whereas 2,6-bis(4chloro phenyl)-1-methylpiperidin-4-one0-(2-(2-(4-nitrobenzylidene) hydrazinyl) thiazol-4-yl)oxime (Fig. 28) exhibited significant antimicrobial activity. Authors mentioned that enhancement in the antioxidant activity of the designed compounds might be due to the radical dissipation activity of thiazole ring and lipophilic nature of piperidinone oxime moieties. It was noticed that active compounds contain the electron donating substituents on the phenyl rings [38].

\section{Piperidine hydrazides}

Sulfonylhydrazones bearing piperidine derivatives (Fig. 29) were synthesized by condensing benzene sulfonylhydrazides with 2 , 6-diphenyl piperidin-4-one and ethyl 4-oxopiperidine-1-carboxylate. Antioxidant and anticholinesterase activity of the compounds was evaluated, and their structural activity relationship was investigated.

Compound possessing methoxy derivative showed good antioxidant activity in CUPRAC assay. Compound substituted with bromo group showed highest antioxidant activity in DPPH, linoleic acid assays and exhibited potent activity in anticholinesterase inhibitory activity [39].

\section{Miscellaneous}

Imine substituted hindered piperidine stabilizers (Fig. 30) were synthesized, and their thermal and light stabilizing action was compared. The imine stabilizer structure is based on 2- hydroxy benzophenone and a 4-amino-tetra methyl piperidine structure. The polymer oxidation rate was determined by carbonyl index using Fourier-transform infrared spectroscopy (Fourier-transform infrared spectroscopy), and its hydroperoxide formation was measured. Most of the compounds were highly effective thermal and light stabilizers for polyolefin films and also inhibited the formation of hydroperoxide during thermal aging in polymers [40].

Plant-derived and synthetic piperidines are extremely important for their antioxidant properties [41-43]. For example, naturally occurred piperidine alkaloids demonstrated promising bactericidal, anticancer, and antioxidant properties [44]. The marked relationship between antioxidant properties and life-threatening diseases such as cancer highlight the significance of piperidines in the current drug research.

\section{AUTHOR'S CONTRIBUTIONS}

Manjusha RK and Shaheen Begum collected the articles and drafted the manuscript. Arifa Begum reviewed and drafted the article. Bharathi $\mathrm{K}$ supervised the review work. All authors discussed and finalized the manuscript.

\section{CONFLICTS OF INTEREST}

None.

\section{REFERENCES}

1. Kumar D, Singh V. Study of heterocyclic compound piperidine. Int J Res Sci Tech 2014; 3:25-8

2. Przedborski S, Jackson-Lewis V, Djaldetti R, Liberatore G, Vila M, Vukosavic S, et al. The parkinsonian toxin MPTP: Action and mechanism. Restor Neurol Neurosci 2000;16:135-42.

3. Aridoss G, Parthiban P, Ramachandran R, Prakash M, Kabilan S, Jeong YT, et al. Synthesis and spectral characterization of a new class of $\mathrm{N}$-(N-methylpiperazinoacetyl)-2,6-diarylpiperidin-4-ones: Antimicrobial, analgesic and antipyretic studies. Eur J Med Chem 2009;44:577-92.

4. Prashanth MK, Revanasiddappa HD, Lokanatha Rai KM, Veeresh B. Synthesis, characterization, antidepressant and antioxidant activity of novel piperamides bearing piperidine and piperazine analogues. Bioorg Med Chem Lett 2012;22:7065-70.

5. Kiasalari Z, Khalili M, Roghani M, Ahmadi A, Mireie M. Antioxidant and antiepileptic activity of 1-[1-(3-Methoxyphenyl) (Tetralyl)] piperidine as a new derivative of phencyclidine on pentylentetrazoleinduced kindling mice. Iran J Pathol 2013;9:138-48.

6. Paulrasu K, Arul D, Manikandan P, Amirthaganesan S, Kuppusamy M, Balasankar T, et al. Synthesis of 4-methyl- N'-(3-alkyl-2r,6C-diaryl piperidin-4-ylidene) -1,2,3-thiadiazole-5-carbohydrazides with antioxidant, antitumor and antimicrobial activities. Org Biomol Chem 2014;12:5911-21.

7. Frietas RM, Silva FO, Silva MG, Feng D. Antioxidant mechanisms of iso-cassine in suppressing seizures induced by pilocarpine. Braz $\mathrm{J}$ Pharmacog 2011;21:437-43.

8. Parthiban P, Aridoss G, Rathika P, Ramkumar V, Kabilan S. Synthesis, stereochemistry and antimicrobial studies of novel oxime ethers of aza/ diazabicycles. Bioorg Med Chem Lett 2009;19:6981-5.

9. Baumann M, Baxendale IR. An overview of the synthetic routes to the best selling drugs containing 6-membered heterocycles. Beilstein J Org Chem 2013;9:2265-319.

10. Bendary E, Francis RR, Ali HM, Sarwat MI, El Hady S. Antioxidant and structure-activity relationships (SARs) of some phenolic and anilines compounds. Ann Agric Sci 2013;58:173-81.

11. Hajhashemi V, Vaseghi G, Pourfarzam M, Abdollahi A. Are antioxidants helpful for disease prevention? Res Pharm Sci 2010;5:1-8.

12. Marambaud P, Zhao H, Davies P. Resveratrol promotes clearance of Alzheimer's disease amyloid-beta peptides. J Biol Chem 2005;280:37377-82.

13. Moon JK, Shibamoto T. Antioxidant assays for plant and food components. J Agric Food Chem 2009;57:1655-66.

14. Nakatani $\mathrm{N}$, Inatani $\mathrm{R}$, Ohta $\mathrm{H}$, Nishioka A. Chemical constituents of peppers (Piper spp.) and application to food preservation: Naturally occurring antioxidative compounds. Environ Health Perspect 
1986;67:135-42

15. Trnka J, Blaikie FH, Logan A, Smith RA, Murphy MP. Antioxidant properties of Mito TEMPOL and its hydroxylamine. Free Rad Res 2009;43:4-12.

16. Kim JH, Shyam PK, Kim MJ, Lee HJ, Lee JT, Jang HY, et al. Enantioselective synthesis and antioxidant activity of 3,4,5-substituted piperidine derivatives. Bioorg Med Chem Lett 2016;26:3119-21.

17. Elanchezhian B, Manivannan N, Selvanathan G. Synthesis, spectral characterization and antioxidant activities of 3'-Methyl-2',6'-diphenyl1,3-dihydrospiro[benzo[D]imidazole-2,4'-piperidine]. Int $\mathrm{J}$ Pharm Chem Biol Sci 2014;4:620-7.

18. Alexidis AN, Rekka EA, Demopoulos VJ, Kourounakis PN. Novel 1, 4 substituted piperidine derivatives. Synthesis and correlation of antioxidant activity with structure and lipophilicity. J Pharm Pharmacol 1995;47:131-7.

19. Kumar KA, Rai KM, Kumar G, Mylarappa BN. A facile route for the synthesis of ethyl, N-aryl-2,6-dioxo-piperid-3-ene-4-carboxylates and their biological activity. Int J Pharm Pharm Sci 2012;4:564-8.

20. Manohar S, Thakur A, Bhatia R, Walia S, Ponnan P, Rawat DS, et al. Antibacterial and antioxidant activity evaluation of novel symmetrical and unsymmetrical $\mathrm{C} 5$-curcuminoids. Indian $\mathrm{J}$ Chem 2015;54B:1235-46.

21. Khalid H, Rehman AU, Abbasi MA, Khan KM. Synthesis, spectral characterization and structure-activity relationship studies on some sulfonamides bearing piperidine nucleus. Int J Pharm Pharm Sci 2012;4:443-8

22. Jahan S, Akhtar S, Kamil A, Saify ZS, Mushtaq N, Arif M, et al. Antibacterial, antifungal and antioxidant activities of derivatives of alkyl piperidine. FUUAST J Biol 2012;2:29-35

23. Bandgar BP, Patil SA, Totre JV, Korbad BL, Gacche RN, Hote BS, et al. Synthesis and biological evaluation of nitrogen-containing benzophenone analogues as TNF-alpha and IL-6 inhibitors with antioxidant activity. Bioorg Med Chem Lett 2010;20:2292-6.

24. Prachayasittikul S, Worachartcheewan A, Lawung R, Ruchirawat S, Prachayasittikul V. Activities of thiotetrahydropyridines as antioxidant and antimicrobial agents. EXCLI J 2009;8:107-14.

25. Naicker L, Venugopala KN, Shode F, Odhav B. Antimicrobial and antioxidant activities of piperidine derivatives. Afr J Pharm Pharmacol 2015;9:783-92.

26. Gangadhara S, Prasad C, Venkateswarlu P. Synthesis, antimicrobial and antioxidant activity of piperidine analog containing transcinnamamides. Indo Am J Pharm Res 2015;5:1-8.

27. Karthik CS, Mallesha L, Mallu P. Synthesis and in vitro biological activity of [1-(Substituted-benzoyl)-piperidin-4-yl]-(2, 4- difluorophenyl)-methanoneoximes. Int J Chem Environ Biol Sci 2015;3:169-74.

28. Chamarthi N, Sudhamani H, Basha SK, Nagam V, Tartte V. Synthesis and characterization of new thiourea and urea derivatives of 6-fluoro3-(piperidin-4-yl) benzo[d]isoxazole: In vitro antimicrobial and antioxidant activity. J Chem Sci 2015;127:1739-46.

29. Shivaprasad CM, Jagadish S, Swaroop TR, Mohan CD, Roopashree R, Rangappa KS, et al. New synthetic benzisoxazole deriatives as antimicrobial, antioxidant and antiinflammatory agents. Eur J Chem
2013;4:402-7

30. Shivaprasad CM, Jagadish S, Swaroop TR, Mohan CD, Roopashree R, Rangappa KS, et al. Synthesis of new benzisoxazole derivatives and their antimicrobial, antioxidant and anti-inflammatory activities. Eur J Chem 2014;5:91-5.

31. Kumar KA, Pavithra G, Renuka N, Kumar GV. Piperidone analogs: Synthesis and their diverse biological applications. Int Res J Pharm App Sci 2012;2:145-54

32. Nithya P, Madhavi C. Antioxidant activity of 3-arylidene-4-piperidones in the 1,1-diphenyl-2-picrylhydrazyl scavenging assay. J Taibah Univ Sci 2017;11:40-5.

33. Karthik N, Nithiya S, Jayabharathi J. Novel piperidone derivatives: Synthesis, spectral and evaluation of antioxidant activity. Int J Drug Dev Res 2011;3:122-7.

34. Premalatha B, Elavarasan S, Bhakiaraj D, Chellakili B, Gopalakrishnan M. Synthesis, antimicrobial and antioxidant activity of novel 2,6-diphenyl-1-3-alkylpiperidin-4-one-O-[2,4,6-tritertiarybutylcyclohexa-2,5-dienon-4-yl]oximes. J Appl Chem 2013;2:1509-15.

35. Tharini K, Sangeetha P. Antioxidant and anti-inflammatory activity of 3,3-dimethyl 2,6- dimethyl piperidine4-one oxime. Int J Chem Sci 2015; $13: 1794-804$

36. Harini ST, Kumar HV, Peethambar SK, Rangaswamy J, Naik N. Novel 2,6-bis (4-methoxyphenyl)-1- methyl piperidin-4-one oxime esters: Synthesis and a new insight into their antioxidant and antimicrobial potential. Med Chem Res 2014;23:1887-98.

37. Harini ST, Kumar HV, Rangaswamy J, Naik N. Synthesis, antioxidant and antimicrobial activity of novel vanillin derived piperidin-4-one oxime esters: Preponderant role of the phenyl ester substituents on the piperidin-4-one oxime core. Bioorg Med Chem Lett 2012;22:7588-92.

38. Harini ST, Kumar HV, Rangaswamy J, Naik N. Synthesis of thiazolebased substituted piperidinone oximes: Profiling of antioxidant and antimicrobial activity. Russ J Bioorg Chem 2017;43:186-96.

39. Karaman N, Oruc-Emre EE, Sicak Y, Catıkkas B, KarakucukA, Ozturk M, et al. Microwave-assisted synthesis of new sulfonylhydrazones, screening of biological activities and investigation of structure-activity relationship. Med Chem Res 2016;25:1590-607.

40. Allen NS, Ortiz RA, Anderson GJ. Comparison of the thermal and light stabilising action of novel imine and piperazine based hindered piperidine stabilisers in polyolefins. Polym Degrad Stab 1994;46:85-91.

41. Vasavirama K, Upender M. Piperine: A valuable alkaloid from Piper species. Int J Pharm Pharm Sci 2014;6:34-8.

42. KumarKS, Mohan CD, JagadishS, RakeshKS, HanumappaA, BasappaB, et al. Synthesis and acetylcholinesterase/butyrylcholinesterase inhibition activity of arecoline-, 4-thiazolidinone- and piperidine-based conjugates. Asian J Pharm Clin Res 2015;8:142-8.

43. Perumal P, Sivakkumar T, Kannappan N, Manavalan R. Synthesis, spectroscopic characterization and antimicrobial activity of $2,6 \mathrm{di}$ substituted piperidine-4-one derivatives. Int J Pharm Pharm Sci 2013; 5 suppl2:317-21

44. Rajbir K, Saroj A. Alkaloids-important therapeutic secondary metabolites of plant origin. J Citation Rep 2015;2:1-8 